\title{
Research on the Application of Smart Helmet and Smart Watch in Power Scene Safety Monitoring
}

\author{
Yusong Guo ${ }^{\mathrm{a}}$, Zhen $\mathrm{Yu}^{\mathrm{b}}$ and Weimei Zhao ${ }^{\mathrm{c}}$ \\ State Grid Smart Grid Research Institute, Beijing 102209, China. \\ aguoyusong@sgri.sgcc.com.cn, byuzhen@sgri.sgcc.com.cn, 'zhaoweimei@sgri.sgcc.com.cn
}

Keywords: smart helmet, smart watch, power scene, safety monitoring.

\begin{abstract}
In order to satisfy the requirements of power operation scene safety monitoring, and lessen the human accidents, wearable technology was applied to power operation scene, and a wearable safety monitoring platform was presented. This paper illustrated the solution of the platform, the design of smart helmet and smart watch, the architecture of backstage monitoring system. The wearable devices like smart helmet and smart watch monitor the scene situation real time, and then transfer the data collected to monitoring system. According to the scene data, the monitoring system makes the judge of operators are in danger or not, and sends out alarm to unsafe operators via wearable devices. Emergency rescue can be carried out timely when accident happens.
\end{abstract}

\section{Introduction}

Safety is the eternal theme of power enterprise. Weak safety mentality, short safety skill, common illegal habits are the major reasons of injury accidents. Recently, power enterprises have equipped many safety tools such as insulation, individual protection and altitude, but most of them don't have active danger alarm function.

Wearable technology is a dime-size computer system worn on the body, it's a new interactive form, and has features such as carry-on, using easily, liberating hands, wireless data transmission, continuing working[1]. Wearable technology is one of the researching hotspots that high-tech companies rush to research. Now, wearable technology is being researched and applied in some important fields in some degree such as industry, health-care, fire fighting and military. The reference[2] researched the application of wearable technology in enterprise device maintenance operation. The reference[3] researched the application of wearable technology in cardiovascular tele-monitoring. The reference[4] researched the application of audio and video wireless transmission and infrared thermal imaging technology in firemen helmet. The reference[5] researched the application of wearable technology in military operation.

Some power device companies have developed electric-approach alarm helmet and watch, but these devices are not commonly used in power scene because of certain reasons. Now, there are no wearable devices which have functions like danger alarm, audio transmission, camera shooting, accidents alarm, applied in power scene human safety monitoring.

\section{Application Scene}

Typical power operation includes device patrol, device maintenance, accident repair, switching operation, line operation, etc. Wearable devices can make a voice alarm, stop illegal behavior and accident, record operation process, photograph device defects, direct operators remotely, light at night, search device parameter and operating standard, when health condition is abnormal, leaping over safety fence arbitrarily, entering charging interval wrongly, less than safety distance, near electric device temperature is too high, $\mathrm{SF}_{6}$ leakage. It also can send out alarm information to remote monitors. After received alarm information, remote monitors can arrive at the scene in time to dispose the alarm event, according to checking the position of operators through e-map. 


\section{Solution}

Total Design Scheme. Safety monitoring platform includes wearable devices and monitoring system. Wearable devices include smart helmet and smart watch, connecting the monitoring system with wireless communication technology. Using the technology of information, communication and sensing, helmet and electronic watch can realize the functions of person location, voice communication, audio and video recording, lighting, photographing, infrared temperature measuring, electric-approach alarm, $\mathrm{SF}_{6}$ concentration monitoring, vital sign monitoring, tumble automatic alarm, emergency alarm, etc. Bluetooth is used as the communication mode between smart helmet and smart watch. Operator location and data transmission with remote monitoring system depends on Wi-Fi (Wireless Fidelity) inside substation, GPS (Global Positioning System) and 4G (the 4th Generation) outside substation. Fig.1 is the overall design idea of safety monitoring platform.

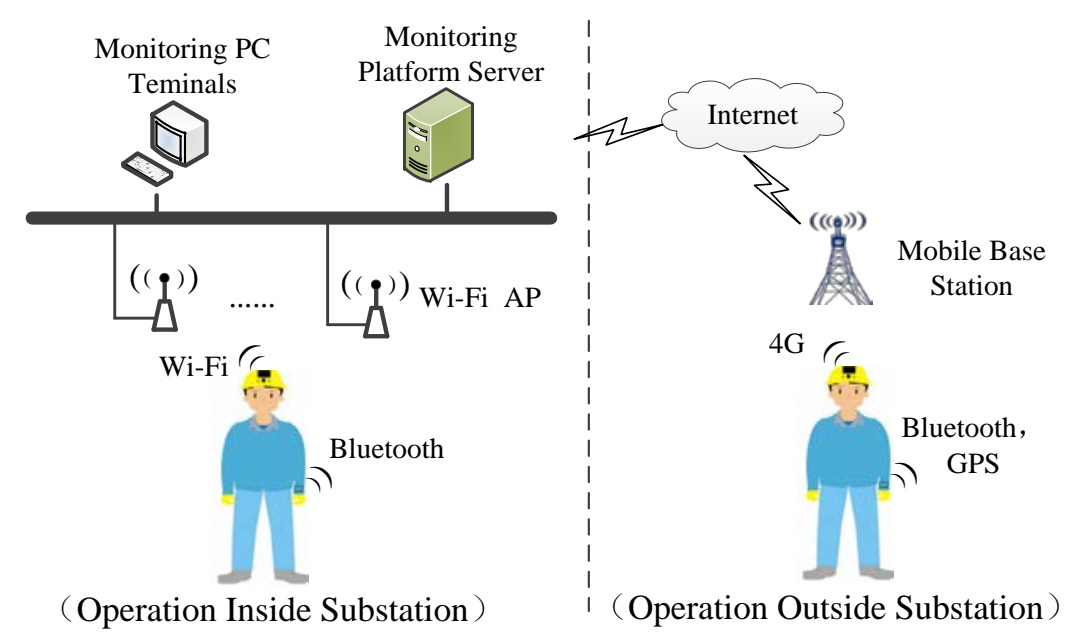

Fig. 1 The overall design idea of safety monitoring platform

Wearable Devices. To reduce the number of buttons on helmet, we use APP software in smart watch to control LED (Light Emitting Diode) light, the self-checking and sensibility adjustment of electric-approach alarm. We can set the names and number of operators before entering the operation scene.

Installed electronic modules on traditional helmet: Wi-Fi, TD-LTE (Time Division Long Term Evolution) 4G, camera, infrared detector, microphone, loudspeaker, Bluetooth, LED light, induction coil of electric-approach alarm, $\mathrm{SF}_{6}$ concentration sensor, infrared temperature sensor, CPU (Central Processing Unit), lithium battery, etc. To ensure the balance of helmet, electronic devices are well-distributed. The function of smart helmet: (1)person location, (2)voice communication, (3)audio and video recording and uploading, (4) photographing, (5) power device infrared temperature measuring, (6)lighting, (7)electric-approach alarming, (8) $\mathrm{SF}_{6}$ leakage alarm, (9)body temperature monitoring.

Installed electronic modules on smart watch: Wi-Fi, GSM (Global System for Mobile Communications), GPRS(General Packet Radio Service), GPS, pulse sensor, Bluetooth, microphone, loudspeaker, alarm button, acceleration sensor, CPU, lithium battery, etc. The function of smart watch: (1)telephone,(2)person location, (3)video and picture displaying, (4handwriting input, (5)data inquiry such as power device parameter and operating standard, 6)vital sign monitoring such as blood pressure and pulse, (7)tumble automatic alarming, (8)emergency manual alarm.

Monitoring System. Monitoring system receives the data that wearable terminals upload, mastering the working mode of all operators, and feedback information to operators. The function of monitoring system: (1)person location, giving out alarm to the operator who is entering charging interval wrongly, (2)database such as power device parameter and operating standard, sending data to wearable terminals according to their application, (3)voice communication with wearable terminals, (4)receiving and displaying the audio and video which wearable terminals recorded, (5)receiving and 
displaying the pictures which wearable terminals photographed, (6)receiving and displaying the infrared thermography which wearable terminals send out, (7)health checking and alarming, (8) receiving the information such as electric-approach alarm, $\mathrm{SF}_{6}$ leakage alarm, tumble alarm, emergency alarm which wearable terminals send out, (9) operation process playback, (10) data statistics and analysis.

Communication Scheme. The communication between smart helmet and smart watch is Bluetooth 4.0 technology which can transmit the data and control signal such as switching LED light, adjusting brightness, self-checking and sensibility adjustment of electric-approach alarm, videotaping, photographing, infrared thermography, etc.

We install one Wi-Fi AP (Access Point) respectively at the center of each wall of the substation, and install another one at the center of the device area in the substation. Smart helmet and smart watch connects the APs wirelessly through Wi-Fi signals. The location accuracy of GPS is low, and its anti-interference capability is poor. So, GPS can't be applied to the complex environment of substation. Using the five APs, Wi-Fi module on helmet and the two Location Algorithm based on RSSI (Received Signal Strength Indication), person location accuracy can reach about $2.7 \mathrm{~m}^{[6]}$, satisfying the basic need of monitoring the patrol path and avoiding entering charging interval wrongly.

The communication between operators and monitoring system depends on 4G module installed on smart helmet, when operating on the power line far from substation. The data transmission of smart watch is transferred by Bluetooth from smart helmet. As monitoring patrol path and avoiding entering charging interval wrongly are not necessary, person location accuracy can be lower than inside substation, using GPS is OK.

\section{Wearable Terminals Hardware Design}

Smart Helmet. Fig.2 is the hardware architecture of smart helmet.

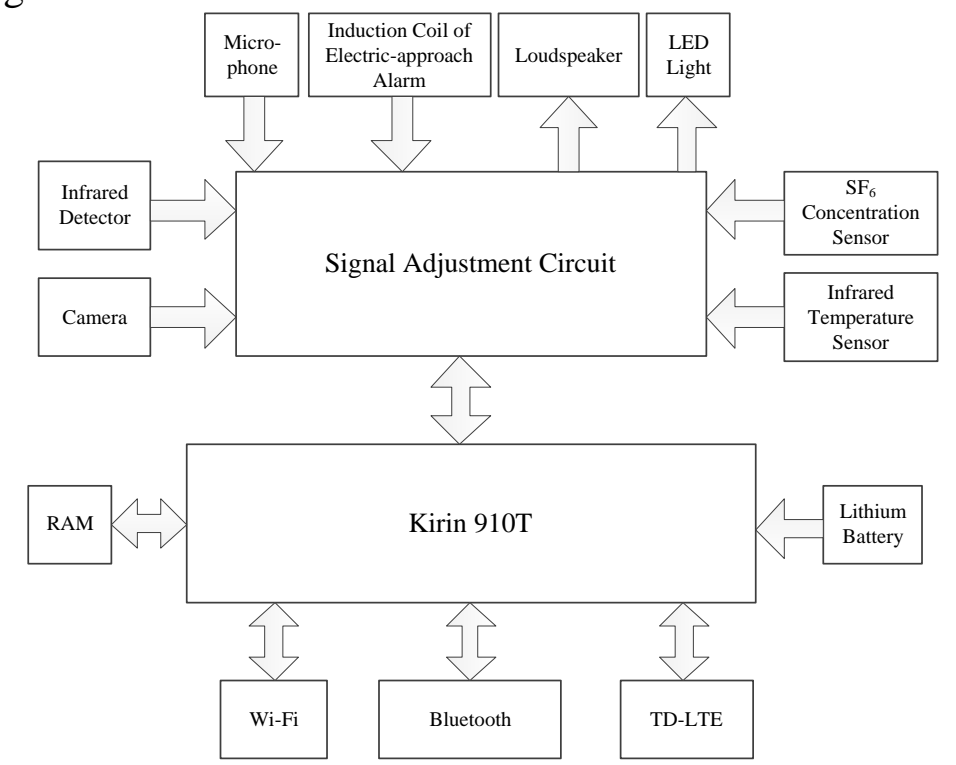

Fig.2 The hardware architecture of smart helmet

The CPU is Huawei Kirin 910T, Quad core, 1.8GHz. The capacity of RAM (Random Access Memory) is 1GB, supporting China Telecom TD-LTE 4G network.

The electric-approach alarm has five sensibility gears, including $10 \mathrm{kV}, 35 \mathrm{kV}, 110 \mathrm{kV}, 220 \mathrm{kV}$, $500 \mathrm{kV}$. Five tunable resistors connect with five sensibility gears individually. The adaptive adjustment of different voltage class depends on APP software in smart watch.

Using the checking method of single channel acoustic attenuation, we can realize the miniaturization and low power consumption of $\mathrm{SF}_{6}$ concentration checking device. $\mathrm{SF}_{6}$ concentration sensor includes ultrasonic transducer, humiture sensor, checking channel, etc ${ }^{[7]}$. 
IR(Infrared Radiation) camera uses uncooled $\mathrm{VO}_{\mathrm{x}}$ infrared focal plane detector, MEMS technology, double layer structure, metal pipe vacuum encapsulation, pixel dimension is $20 \mu \mathrm{m}$, array is $640 \times 512$, NETD (Noise Equivalent Temperature Difference) is $40 \mathrm{mK}$, thermal response time is $20 \mathrm{~ms}$, response rate is $12 \mathrm{mV} / \mathrm{K}$, response range is $8 \sim 16 \mu \mathrm{m}^{[8]}$.

GE ZTP135S-R sensor is applied to measure forehead temperature. Its structure has smaller thermal inertia and higher sensibility. The environment temperature compensation depends on negative temperature ratio electric heating adjuster ${ }^{[9]}$.

Smart Watch. Fig.3 is the hardware architecture of smart watch.

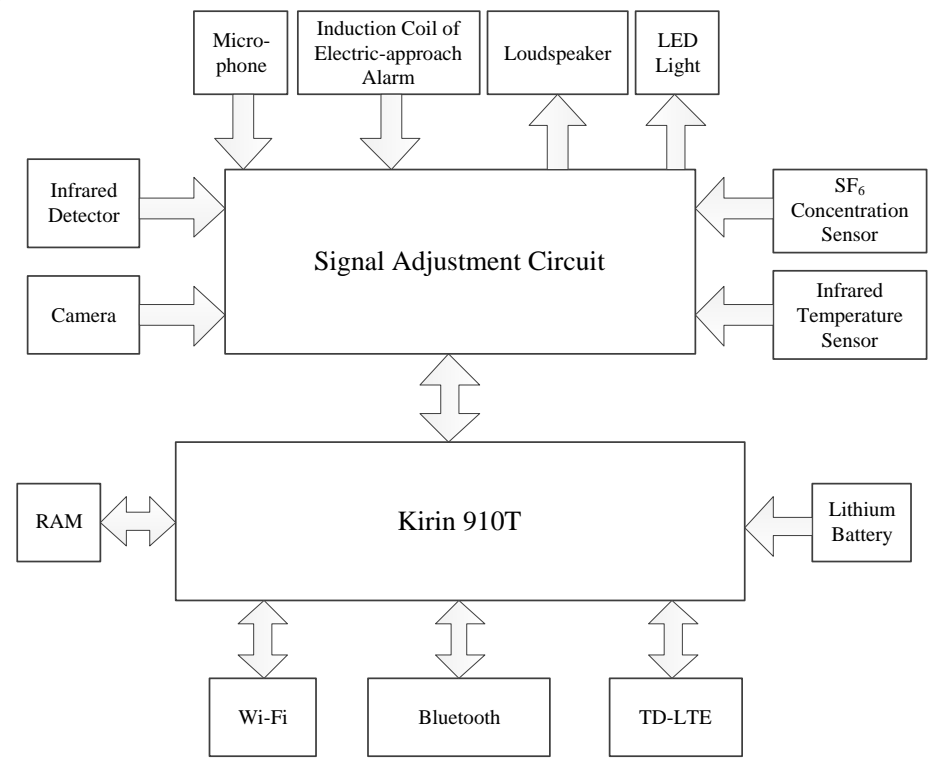

Fig.3 The hardware architecture of smart watch

Smart watch uses Android 4.3 operating system, screen inch is 1.63, resolution ratio is $320 \times 320$, touch-screen operation, handwriting input. The CPU is MT6589, Quad core, 1.2GHz. The capacity of RAM is $512 M B$, ROM (Read Only Memory) is 4GB.

The pulse sensor based on piezoelectricity theory ${ }^{[3]}$ is fixed in watchband, connecting with the signal adjustment circuit in watch through two leads. The size of detection head is $15 \mathrm{~mm} \times 3 \mathrm{~mm}$, pressure range is $5.8 \sim 11.6 \mathrm{PSI}$ (Pounds per Square Inch). The interface of the sensor connects with wrist artery, converting the pulse signals to voltage signals.

The acceleration sensor uses Freescale MMA7260Q tri-axis accelerometer, the denoising method of sensor data is Kalman filtering algorithm. Posture angle is the tumble judgment standard, and it has a high tumble detection accuracy ${ }^{[10]}$.

Emergency alarm is designed as solid button, fixed at one side of the watch.

\section{Monitoring System Design}

Technology Architecture. The architecture of monitoring system is B/S (Browser/Server). Data expression and voice communication are showed at the browser. Affair logic and database management are realized at the server. The overall function frame is the layering design idea, and it is divided as basic device layer, supporting layer, business layer and expression layer. Fig.4 is the specific content of the overall function frame.

Basic device layer includes location device (Wi-Fi AP), network device (router, switch, firewall), server. Supporting layer is the bottom basic software such as data collection, processing and storage, including TCP/IP (Transmission Control Protocol/Internet Protocol), RTSP (Real Time Streaming Protocol), location algorithm, e-map, database, etc. Business layer is the function of power operation safety monitoring, including entering charging interval alarm, data inquiry, voice communication with operators, displaying the audio and video of the scene timely, displaying the picture of the scene, displaying the infrared thermography of the scene, health alarm, receiving danger alarm signals, operation process playback, history information searching and statistics. Expression layer is the 
output and displaying of the monitoring and alarm information, including monitoring PC terminals, audio terminals, smart helmet, smart watch.

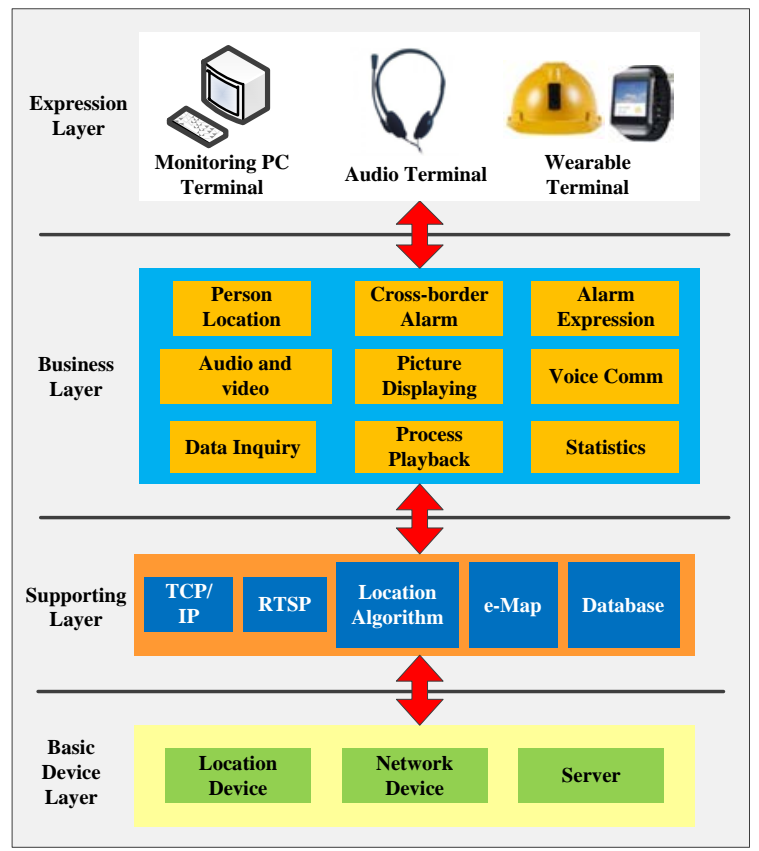

Fig.4 The overall function frame

Person Location. Substation e-map is a 2D map. Monitoring system displays the movement trail of operators according to the coordinates of operators received from Wi-Fi location subsystem. Monitoring system judges operators crossing boarder or not through contrasting the coordinates of operators and the coordinates of operation area and electric area. Monitoring system sends out alarm to the operator in time if he is crossing the boarder. The corresponding operator is displayed as a red sign in e-map, and the alarm information is listed at the same time, when monitoring system sending out alarm or receiving alarm from wearable terminals.

A constructed GIS (Geographic Information System) or Baidu map is needed when operating outside substation, it displays the location of operators according to GPS coordinates.

Alarm Recording and Searching. The alarm affair list is set in the monitoring system. The alarm information is displayed in e-map and alarm affair list at the same time, and then is stored in database. The alarm content includes operator name, alarm type, alarm time, alarm site. We can search and playback the information stored in monitoring system such as alarm content, operator movement trail, audio and video, picture, infrared thermography, when investigating accidents. Monitoring system also has the function of statistics and analysis, which can count the times of operation, operators, alarm, alarm type in a period of time, through the combination of date, team name, substation name, person name, operation type and alarm type.

\section{Summary}

The time of miniaturized computer has come. This paper can partly solve the problem of power operation safety monitoring, and increase work efficiency, through applying wearable technology to power operation scene. Its application field and prospect is very wide. Now, the research of wearable technology applied in power operation is just beginning. So, some problems not easily solved are inevitable, such as heavy, low endurance power, poor sensitivity and costly price. But these problems are being solved in the future along with the rapid development of wearable technology. Wearable devices such as smart helmet and smart watch used in power and other industry are becoming increasingly popular in the future. 


\section{References}

[1] D.Y.Chen, The evolution and trend of wearable computer(I), Journal of Chongqing University(Natural Science Edition), 23(2000)119-124.

[2] S.J.Xiahou, D.Y.Chen, Z.Q.Huang, On-site task assistant pattern based on wearable computing , Computer Integrated Manufacturing Systems, 13(2007) 2269-2275.

[3] J.N.Xiang, S.B.Lu, Mobile and wearable technology in the cardiovascular area of tele-monitoring application, Journal of University of Electronic Science and Technology of China, 39 Suppl. (2010) $1-5$.

[4] L.Zhang, Z.G.Xu, Research and development of firemen multi-functional audio and video communication helmet device, China Public Security·Academy Edition, 23(2011)68-71.

[5] G.Zhang, J.W.Zheng, T.H.Wu, The application of wearable technology in the field of military activity, Beijing Biomedical Engineering, 28(2009) 210-214.

[6] L.B.Pan, Rearch and implementation of wireless location algorithm based on Wi-Fi, MS., Zhejiang University, China, (2013 )39-50.

[7] S.Y.Yan, M.L.Shan, C.P.Zhu, Q.B.Han, Sulfur hexafluoride wireless sensor based on acoustic attenuation method, Piezoelectrics \& Acoustooptics, 35(2013) 698-701.

[8] S.Y.Lei, H.Fang, J.Liu, X.He, Research on domestic 640×512 uncooled VOx infrared focal plane array infrared technology, 35(2013) 759-763.

[9] G.L.Peng, Z.G.Liu, L.Liu, Application of ZTP135S-R sensor in the thermometer, International Electronic Elements, 4 (2006) 38-40.

[10] R.Wang, Y.Zhang, J.X.Chen, Design and implementation of fall detection system using tri-axis accelerometer, Journal of Computer Applications, 32(2012) 1450-1452. 